Published in final edited form as:

Adv Mater. 2020 February ; 32(8): e1906022. doi:10.1002/adma.201906022.

\title{
In vivo sequestration of innate small molecules to promote bone healing
}

\author{
Yuze Zeng ${ }^{1,2}$, Yu-Ru V. Shih ${ }^{1}$, Gurpreet S. Baht ${ }^{1,3}$, Shyni Varghese ${ }^{1,2,4,{ }^{*}}$ \\ ${ }^{1}$ Department of Orthopaedic Surgery, Duke University School of Medicine, Durham, NC 27710, \\ USA \\ ${ }^{2}$ Department of Mechanical Engineering and Materials Science, Duke University, Durham, NC \\ 27710, USA \\ ${ }^{3}$ Duke Molecular Physiology Institute, Durham, NC 27701, USA \\ ${ }^{4}$ Department of Biomedical Engineering, Duke University, Durham, NC 27710, USA
}

\begin{abstract}
Approaches that enable innate repair mechanisms hold great potential for tissue repair. Herein, we describe biomaterial-assisted sequestration of small molecules to localize pro-regenerative signaling at the injury site. Specifically, we designed a synthetic biomaterial containing boronate molecules to sequester adenosine, a small molecule ubiquitously present in the human body. The biomaterial-assisted sequestration of adenosine leverages the transient surge of extracellular adenosine following injury to prolong local adenosine signaling. We demonstrated that implantation of the biomaterial patch following injury establishes an in-situ stockpile of adenosine, resulting in accelerated healing by promoting both osteoblastogenesis and angiogenesis. The adenosine content within the patch recedes to the physiological level as the tissue regenerates. In addition to sequestering endogenous adenosine, the biomaterial is also able to deliver exogenous adenosine to the site of injury, offering a versatile solution to utilizing adenosine as a potential therapeutic for tissue repair.
\end{abstract}

\section{Keywords}

Adenosine; biomaterial; sequestration; delivery; bone healing

\begin{abstract}
A leading concept in regenerative medicine is transplantation of tissue-specific cells, often supported by biomaterials, to promote tissue repair. While this strategy has achieved some success, its broad clinical application is hindered by various challenges such as high costs, constraints associated with cell isolation and expansion, and limited in vivo engraftment of
\end{abstract}

\footnotetext{
"Corresponding Author: To whom Correspondence should be addressed. shyni.varghese@ duke.edu, Tel: +1-919-660-5273. Author contributions

Y.Z. and S.V. designed the study and wrote the manuscript. Y.Z. executed the study and organized the results. G.S.B and Y.V.S. conducted the animal surgeries. Y.V.S. contributed to overseeing the experiments and interpreting the data.

The authors declare no competing financial interest.

Associated content

Supporting Information
} 
transplanted cells ${ }^{[1]}$. Instead, mobilizing endogenous cells to augment the innate regenerative ability of tissues has been explored as an alternative ${ }^{[2]}$. Given that the function of endogenous cells is regulated by their microenvironment, potential of biomaterials and/or growth factors to create pro-healing niches for endogenous cells has been explored extensively ${ }^{[2 b, 3]}$. Meanwhile, naturally-occurring small molecules are also appealing and equally powerful in regulating various cellular functions including tissue-specific differentiation of stem cells ${ }^{[4]}$. Although significant strides have been made in employing small molecules to direct cellular functions in vitro, harnessing small molecules towards tissue repair in vivo still remains limited.

In this study, we determine whether sequestration of small molecules could be used to augment endogenous cell function leading to improved tissue repair. Adenosine is a small molecule ubiquitously present in the human body which acts as an extracellular signaling molecule through G-protein coupled adenosine receptors ${ }^{[5]}$. While the physiological concentration of extracellular adenosine is often insufficient to activate adenosine receptors ${ }^{[6]}$, an increase in extracellular adenosine is observed following tissue injury, which is integral to the natural repair mechanism ${ }^{[5 a, 7]}$. However, this increase is transient as adenosine is rapidly metabolized ${ }^{[8]}$. Although delivery of adenosine can be employed to activate adenosine signaling and address tissue dysfunctions, in practice, such an approach has remained elusive. This is mainly due to the ubiquitous nature of adenosine and the potential off-target effects associated with its systemic administration ${ }^{[8 b, 9]}$. Instead, approaches that localize adenosine signaling at the targeted tissue site can circumvent these limitations and open up new viable therapeutic strategies.

To this end, we have developed a biomaterial-based approach to sequester extracellular adenosine capitalizing on its transient surge following trauma or deliver exogenous adenosine to sustain the activation of adenosine signaling strictly at the injury site.

Specifically, we leveraged the ability of boronate molecule to bind to adenosine via dynamic covalent bonding ${ }^{[10]}$ (Fig. 1a). By employing a 3-(acrylamido)phenylboronic acid (PBA)functionalized polyethylene glycol (PEG) network, we demonstrated in vivo sequestration of adenosine and its application to accelerate bone repair in a murine model (Fig. 1b). We used bone fracture as a model, due to its clinical relevance ${ }^{[11]}$. Bone fracture is also well-suited for studying adenosine-mediated tissue repair, as extracellular adenosine and its receptors play a key role in maintaining bone homeostasis and function ${ }^{[6,7 b, 12]}$ and have been proven to induce osteogenic differentiation of progenitor cells ${ }^{[4 c, 13]}$.

\section{Scaffolds functionalized with PBA groups sequester adenosine both in vitro and in vivo}

To examine the PBA-assisted sequestration and release of adenosine, we have created macroporous PEG scaffolds containing varying amounts of PBA $(0,0.5 \mathrm{M}$, and $1 \mathrm{M}$ as in the reaction mixture), termed as $\mathrm{PBA}_{0}, \mathrm{PBA}_{0.5}$, and $\mathrm{PBA}_{1.0}$, respectively. The macroporous PEG scaffolds were developed by using polymethyl methacrylate (PMMA) microspheres as a porogen, resulting in an interconnected macroporous architecture ${ }^{[14]}$ (Fig. S1, Supporting Information). UV/vis analysis of the residual PBA in the reaction mixture and nuclear 
magnetic resonance (NMR) spectra of the resulting scaffolds suggest more than $90 \%$ of the PBA molecules were reacted and incorporated into the network (Table S1 and Fig. S2, Supporting Information). To determine PBA-mediated adenosine sequestration, the macroporous scaffolds with different levels of PBA were incubated in an excess adenosine solution $(6 \mathrm{mg} / \mathrm{mL}$ in PBS) for $6 \mathrm{~h}$ and the bound adenosine was measured using UV/vis spectroscopy. As shown in Fig. 2a and Fig. S3a (Supporting Information), the amount of sequestered adenosine increased as the amount of PBA within the scaffold increased. Specifically, the $\mathrm{PBA}_{1.0}$ scaffolds had a sequestration efficiency (the amount of PBA moieties involved in adenosine binding) of $75 \%$ with a loading capacity (weight percentage of adenosine in the scaffold) of $28 \%$, while those of the $\mathrm{PBA}_{0.5}$ scaffolds were $59 \%$ and $11 \%$, respectively (Fig. 2a). On the contrary, the PEG scaffolds without PBA moieties (i.e. $\mathrm{PBA}_{0}$ ) had no detectable adenosine content, suggesting that the loading of adenosine was primarily due to the PBA moieties. Since the $\mathrm{PBA}_{1.0}$ scaffolds sequestered more adenosine compared to $\mathrm{PBA}_{0.5}$, they were used for the rest of the studies. The release of adenosine was tested by incubating the $\mathrm{PBA}_{1.0}$ scaffolds in a cell culture medium depleted of nucleosides, which showed a robust release during the first $10 \mathrm{~d}$ followed by a plateau (Fig. 2b).

Having established the ability of PBA scaffolds to sequester and release adenosine in vitro, we next investigated their potential to sequester adenosine in vivo. The ability of $\mathrm{PBA}_{1.0}$ scaffolds to sequester adenosine in vivo was first assessed by using a subcutaneous model. Roughly, $600 \mu \mathrm{L}$ of sterile saline solution containing varying amounts of adenosine $(0,0.25$ or $0.5 \mathrm{mg} / \mathrm{mL}$ ) was injected into an area adjacent to the scaffolds, which had been implanted subcutaneously into mice for $1 \mathrm{~d}$. The scaffolds were retrieved within $1 \mathrm{~h}$ and analyzed for the sequestered adenosine. As anticipated, the $\mathrm{PBA}_{1.0}$ scaffolds retrieved from the cohort injected with $0.5 \mathrm{mg} / \mathrm{mL}$ adenosine had higher adenosine content compared to that received $0.25 \mathrm{mg} / \mathrm{mL}$ adenosine or saline alone (Fig. $2 \mathrm{c}$ and Fig. S3b, Supporting Information). The $\mathrm{PBA}_{1.0}$ scaffolds from the cohort that received only the saline injection were also positive for adenosine, albeit a small amount, which is attributed to the endogenous adenosine present at the site of implantation. On the contrary, no adenosine was detected in the $\mathrm{PBA}_{0}$ scaffolds, further corroborating the necessity of PBA moieties for adenosine sequestration.

Although the physiological extracellular adenosine concentration in most organs is low, its level in the extracellular milieu is known to increase following trauma or injury [7a, 7b]. Consistent with the existing knowledge, our time-dependent analyses of extracellular adenosine following unilateral tibial fracture of mouse showed a significant increase in the adenosine level at the injury site compared to that at the non-fractured contralateral site (Fig. S4, Supporting Information). A roughly 10-fold increase in extracellular adenosine was observed within $1 \mathrm{~d}$ following the injury. To determine the ability of PBA scaffolds to sequester extracellular adenosine by leveraging its surge following fracture, scaffolds were implanted at tibial fracture site upon injury and excised after $3 \mathrm{~d}$. Compared to the $\mathrm{PBA}_{0}$ scaffolds retrieved from the fracture site, those as-retrieved $\mathrm{PBA}_{1.0}$ scaffolds contained a significantly higher amount of adenosine (Fig. 2d). This increased adenosine content within the implant was found to be diminished to a concentration similar to pre-fracture levels by $21 \mathrm{~d}$ (Fig. S5, Supporting Information). Together, the results suggest that biomaterials containing PBA molecules can be used to sequester and enrich extracellular adenosine locally in response to injury. 


\section{PBA-Adenosine conjugation promotes stem cell osteogenesis both in vitro and in vivo}

The osteoanabolic potential of adenosine bound to the scaffold was examined in vitro in a 3D culture by using human mesenchymal stem cells (hMSCs) as a cell source. Towards this, macroporous scaffolds with and without adenosine ( $\mathrm{PBA}_{1.0}-\mathrm{ADO}$ and $\mathrm{PBA}_{1.0}$, respectively) were loaded with hMSCs and cultured in growth medium (GM). Cell-laden PBA 1.0 scaffolds cultured in osteogenic-inducing medium (OM) were used as a positive control. We have previously shown that macroporous scaffolds with an interconnected macroporous structure can facilitate infiltration of the loaded cells, allowing their homogenous distribution within the scaffold ${ }^{[14]}$. Live/Dead assay and PicoGreen DNA assay as a function of culture time showed good cell viability and comparable DNA content in all groups, respectively (Fig. S6, Supporting Information). Osteogenic differentiation of hMSCs in various culture conditions was evaluated through time-resolved quantitative analyses for multiple osteogenic genes osteocalcin (OCN), osteopontin (OPN) and osterix (OSX). As shown in Fig. 3a, the expressions of OCN, OPN, and OSX were consistently up-regulated throughout $21 \mathrm{~d}$ of culture in the $\mathrm{PBA}_{1.0}$-ADO scaffolds similar to the positive control. In contrast, the expressions of osteogenic markers remained low in corresponding cultures with scaffolds lacking adenosine. Consistent with these findings, quantification of calcium content exhibited significantly higher calcium deposition in the $\mathrm{PBA}_{1.0}$-ADO scaffolds compared to the $\mathrm{PBA}_{1.0}$ scaffolds with the same culture condition at the end of $21 \mathrm{~d}$ (Fig. 3b). These results suggest that the sequestered adenosine within the scaffolds promoted osteogenic differentiation of hMSCs akin to cultures involving medium supplemented with $\operatorname{adenosine}^{[4 \mathrm{c}, 13 \mathrm{a}]}$.

We next evaluated the potential of adenosine-bound scaffolds to support in vivo bone formation by adopting an ectopic model ${ }^{[15]}$. Both $\mathrm{PBA}_{1.0}-\mathrm{ADO}$ and $\mathrm{PBA}_{1.0}$ scaffolds loaded $^{2}$ with hMSCs were implanted into the subcutaneous space of immunodeficient mice for $28 \mathrm{~d}$. Upon retrieval, the $\mathrm{PBA}_{1.0}$-ADO scaffolds were found to be opaque (Fig. 3c and Fig. S7, Supporting Information). Radiographs generated from the microcomputed tomography $(\mu \mathrm{CT})$ scans showed a strong optical signal from the $\mathrm{PBA}_{1.0}$-ADO scaffolds, which is consistent with the gross appearance, suggesting in vivo calcification and the presence of hard tissue formation (Fig. 3c). The $3 \mathrm{D}$ rendering of the excised PBA 1.0 -ADO scaffolds showed an even distribution of mineral deposition within the scaffolds, as indicated by both top and oblique views (Fig. 3d). Conversely, the excised $\mathrm{PBA}_{1.0}$ scaffolds did not display this opaque appearance nor apparent calcification. Based on the quantification of $\mu \mathrm{CT}$ results, the $\mathrm{PBA}_{1.0}$-ADO scaffolds had a bone volume ratio (BV/TV) of $14.4 \%$ and a bone mineral density (BMD) of $0.51 \mathrm{~g} / \mathrm{cm}^{3}$, compared to $1.6 \%$ and $0.05 \mathrm{~g} / \mathrm{cm}^{3}$ found within the PBA $_{1.0}$ group (Fig. 3e). Measurement of calcium content within the scaffolds, $97.3 \pm 4.8$ $\mathrm{mg} / \mathrm{g}$ dry weight in the $\mathrm{PBA}_{1.0}$-ADO and $18.2 \pm 0.8 \mathrm{mg} / \mathrm{g}$ dry weight in the $\mathrm{PBA}_{1.0}$ scaffolds (Fig. 3f), further confirmed higher in vivo calcification of the cell-laden $\mathrm{PBA}_{1.0}-\mathrm{ADO}$ scaffolds. Bone tissue formation was further evaluated by histological characterization. Hematoxylin and eosin (H\&E) staining of the excised implants showed dense extracellular matrix (ECM), resembling that of the bone tissue, in the cell-laden $\mathrm{PBA}_{1.0}$-ADO scaffolds, whereas the corresponding $\mathrm{PBA}_{1.0}$ group had minimal bone tissue formation (Fig. $3 \mathrm{~g}$ ). 
Furthermore, positive staining of OCN, an ECM protein secreted by osteoblasts, was seen throughout the $\mathrm{PBA}_{1.0}$-ADO scaffolds (Fig. $3 \mathrm{~g}$ ). Together, the findings suggest that the adenosine-loaded scaffolds supported osteogenic differentiation of the transplanted hMSCs and promoted ectopic bone formation, which further corroborates the osteoblastogenic function of adenosine.

\section{PBA-mediated adenosine sequestration promotes bone fracture healing}

We employed a tibial fracture model to investigate the role of biomaterial-assisted sequestration of adenosine in bone repair, which is a comprehensive process involving cartilaginous callus formation at the injury site, endochondral ossification within the callus, and callus/bone remodeling ${ }^{[16]}$. Stabilized fractures were induced unilaterally at tibial midshafts in mice ${ }^{[17]}$, and biomaterials with uniform dimensions were used to cover the fracture sites. In addition to $\mathrm{PBA}_{0}$ and $\mathrm{PBA}_{1.0}$ patches, we also used patches pre-loaded with exogenous adenosine ( $\left.\mathrm{PBA}_{1.0} \mathrm{ADO}\right)$. We monitored the fracture healing as a function of time using radiographic and histomorphometric analyses.

Figure 4a shows 3D reconstructed images (intact and cut views) and their corresponding radiographs of the injured tibiae during fracture healing. The evolution of callus as bone repair progresses is represented in Figure 4b. At $7 \mathrm{~d}$, the fractures were still evident in all groups owing to the minimal mineralization of the calluses. As time progressed, the calluses calcified, and the extent of mineralization was found to correlate with the type of intervention, where both the $\mathrm{PBA}_{1.0}-\mathrm{ADO}$ and the $\mathrm{PBA}_{1.0}$ groups exhibited a better bridging of the fracture. By $21 \mathrm{~d}$, growing calluses eventually bridged the fracture gaps in all groups. Interestingly, radiographic images at $21 \mathrm{~d}$ showed cortical bridging only in groups treated with $\mathrm{PBA}_{1.0}-\mathrm{ADO}$ and the $\mathrm{PBA}_{1.0}$ (Figure 4a,b), suggesting a faster healing compared to those treated with $\mathrm{PBA}_{0}{ }^{[16]}$. Concomitant with these observations, analysis of the fracture sites at $21 \mathrm{~d}$ from axial view (Figure $4 \mathrm{c}$ ) revealed better remodeled patterns and more organized lamellar bone formation in cohorts that received either $\mathrm{PBA}_{1.0}-\mathrm{ADO}$ or $\mathrm{PBA}_{1.0}$ patches. These findings were further confirmed by the quantification of the $\mu \mathrm{CT}$ scans at 14 $\mathrm{d}$ and $21 \mathrm{~d}$, by which callus ossification was more evident compared to $7 \mathrm{~d}$. By $14 \mathrm{~d}$ (Fig. S8, Supporting Information), bone volume was higher in both the $\mathrm{PBA}_{1.0}-\mathrm{ADO}$ and the $\mathrm{PBA}_{1.0}$ groups, albeit with no statistical significance. The differences in bone formation were apparent by $21 \mathrm{~d}$ (Figure 4d), where the fractures treated with $\mathrm{PBA}_{1.0}-\mathrm{ADO}$ and $\mathrm{PBA}_{1.0}$ exhibited significantly higher bone volume ratio within the calluses compared to those treated with $\mathrm{PBA}_{0}$. Together, the results demonstrate the prevalent role of localized adenosine signaling in promoting callus maturation and fracture healing. When the biomaterial patch was dosed once with exogenous adenosine, as in the case of the $\mathrm{PBA}_{1.0^{-}}$ ADO group, the healing was further improved, mostly due to the higher amount of adenosine available.

Given the importance of the evolution of cartilaginous tissue, vascularization, and osteoclastdriven bone resorption in fracture healing ${ }^{[16]}$, we also examined the effect of biomaterialmediated adenosine signaling on cartilaginous tissue, blood vessel formation, and osteoclast activity during healing. We observed intense cartilage formation (stained red) within the calluses of both the $\mathrm{PBA}_{1.0}-\mathrm{ADO}$ and the $\mathrm{PBA}_{1.0}$ groups at $7 \mathrm{~d}$ (Fig. $4 \mathrm{e}$ ), followed by 
cartilage resorption over time suggesting endochondral ossification. In contrast, the animals treated with the $\mathrm{PBA}_{0}$ showed delayed cartilaginous tissue formation and remodeling. Cartilaginous tissues still remained in the calluses of these animals at $21 \mathrm{~d}$ (Fig. 4e). Concurrent with these findings, an intervention-specific change in vascularization of the calluses was also observed (Fig. 4f-i). Specifically, more endomucin (EMCN)-positive blood vessels were detected in both the $\mathrm{PBA}_{1.0}-\mathrm{ADO}$ and the $\mathrm{PBA}_{1.0}$ groups compared to the $\mathrm{PBA}_{0}$ cohort at $7 \mathrm{~d}$ (Fig. $4 \mathrm{~g}$ ) and $14 \mathrm{~d}$ (Fig. 4h). The improved callus vascularization in the presence of PBA-mediated adenosine signaling may be directly linked to the established role of adenosine in promoting angiogenesis ${ }^{[18]}$. The improved osteoblastogenesis observed in these groups could also contribute to the increased angiogenesis ${ }^{[19]}$. While there were differences in angiogenesis among the different groups at early time points, no interventiondependent differences in blood vessel content were observed at $21 \mathrm{~d}$ (Fig. 4i). Analyses of osteoclast activity via TRAP staining (Fig. S9a, Supporting Information) showed increased TRAP-positive area with time in all the groups. Particularly, higher percentage of TRAPpositive area in the $\mathrm{PBA}_{1.0}$-ADO cohort at $21 \mathrm{~d}$ (Fig. S9b, Supporting Information), indicating higher bone remodeling, which could be associated with high levels of bone formation.

To summarize, the cohorts treated with $\mathrm{PBA}_{1.0}$ or $\mathrm{PBA}_{1.0^{-}} \mathrm{ADO}$ showed a better healing outcome compared to those treated with $\mathrm{PBA}_{0}$, as evidenced by the extent of callus maturation, endochondral calcification, and angiogenesis, suggesting that biomaterialmediated localization of adenosine signaling promotes bone healing. Furthermore, the increased adenosine concentration from the biomaterial-mediated sequestration recedes to the physiological level with healing (Fig. S5, Supporting Information), which underscores the translational potential of the described strategy. While PBA-mediated sequestration of endogenous adenosine alone promoted fracture healing, the augmentation of adenosine level with a one-time supplement of exogenous adenosine (i.e. $\mathrm{PBA}_{1.0}$-ADO) further improved callus vascularization and healing outcome. Note that the $\mathrm{PBA}_{1.0}$ sequestered only a small fraction of the adenosine being released by cells (Fig. 2d), further improvement of biomaterial design, such as increasing PBA content or changing the architecture to increase surface-to-volume ratio, could be used to enhance the sequestration efficiency. Such an approach will imbibe more adenosine from the milieu following injury and sustain its local concentration and could eliminate the need for exogenous adenosine entirely. Nonetheless, the results presented in this study showed the potential of using a PBA-containing biomaterial to boost the adenosine concentration at the fracture site and leverage the natural repair mechanism involving adenosine signaling to promote fracture healing.

This proof-of-concept study demonstrates that sequestration of adenosine, a native small molecule, by biomaterials at the fracture site can be used to promote bone fracture healing. The sequestration and release of adenosine was achieved by harnessing the ability of boronate molecules to form dynamic covalent bonds with cis-diol molecules such as adenosine. This biomaterial approach sustained an elevated concentration of adenosine locally by leveraging the surge of extracellular adenosine following injury and created a proregenerative milieu through localized adenosine signaling, resulting in improved bone repair. Besides sequestering endogenous adenosine, the biomaterial can also be used to deliver exogenous adenosine to the injury site, especially in pathological situations encountering 
diminished extracellular adenosine. By enabling a prolonged adenosine signaling, this biomaterial approach circumvents potential off-target effects associated with the systemic administration of adenosine, which is a major hurdle in harnessing adenosine signaling as a potential therapeutic.

Moving forward, the biomaterial-assisted sequestration of extracellular adenosine can be used to create an in-situ stockpile of the small molecule, which can be conveniently replenished non-invasively through injections. For example, local modulation of the adenosine signaling may be used to prevent repeated fractures, which are commonly observed in the aged population, as well as in patients suffering with osteoporosis and other bone-degenerating diseases. In the future, age- and sex-dependent fracture healing in response to extracellular adenosine can also be examined to determine how the adenosine metabolism may differ and lead to different repair outcomes. The biomaterial can be adapted accordingly to mirror the innate repair mechanism by modulating the extent of adenosine sequestration.

\section{Experimental Section}

\section{Materials}

Polyethylene glycol diacrylate (PEGDA) and N-acryloyl-6-aminocaproic acid (A6ACA) were synthesized as previously described ${ }^{[20]}$. PEG macroporous scaffolds containing the PBA moieties were fabricated using a poly(methyl methacrylate) (PMMA) leaching method $^{[14 a]}$.

\section{Adenosine sequestration and release}

Macroporous scaffolds were soaked in adenosine solution for in vitro loading, and the release of adenosine in cell culture medium was measured by UV/vis. Scaffolds were implanted in subcutaneous region and tibial fracture site for in vivo sequestration, and the adenosine content was quantified using an Adenosine Assay.

\section{Stem cell osteogenesis in PBA scaffolds}

Sterile scaffolds loaded with primary hMSCs were either cultured in vitro or implanted subcutaneously. Osteogenic differentiation was evaluated using PCR, microcomputed tomography, and histology.

\section{Tibial fracture and healing}

All animal studies were conducted with the approval of the Institutional Animal Care and Use Committee (IACUC) at Duke University and complied with NIH guidelines for laboratory animal care. Tibial fracture was induced ${ }^{[17]}$, and biomaterial patches were applied at the fracture sites. Bone healing with time was assessed using microcomputed tomographic and histomorphometric analyses. Details for the experiments are presented in the Supporting Information. 


\section{Supplementary Material}

Refer to Web version on PubMed Central for supplementary material.

\section{Acknowledgments}

We thank Dr. Jiaul Hoque for assisting the NMR characterization. We are grateful to Dr. Matthew Hilton, Dr. Rong Huang, and Anthony Mirando for their help with histomorphometric analysis. This work was partially supported by the National Institute of Arthritis and Musculoskeletal and Skin Diseases of the National Institutes of Health under Award Number NIH R01 AR063184 and AR071552. The content is solely the responsibility of the authors and does not necessarily represent the official views of the National Institutes of Health. The hMSCs were provided by Institute for Regenerative Medicine (Texas A\&M University) through NIH Grant P40RR017447 from the National Center for Research Resources.

\section{References}

[1] a). Segers VFM, Lee RT, Nature 2008, 451, 937; [PubMed: 18288183] b) Salem HK, Thiemermann C, Stem Cells 2010, 28, 585; [PubMed: 19967788] c) Grayson WL, Bunnell BA, Martin E, Frazier T, Hung BP, Gimble JM, Nat. Rev. Endocrinol 2015, 11, 140. [PubMed: 25560703]

[2] a). Dimmeler S, Ding S, Rando TA, Trounson A, Nat. Med. 2014, 20, 814; [PubMed: 25100527] b) Chen FM, Wu LA, Zhang M, Zhang R, Sun HH, Biomaterials 2011, 32, 3189; [PubMed: 21300401] c) Gonzalez Diaz E, Shih Y, Nakasaki M, Liu M, Varghese S, Tissue Engineering 2018;d) Phinney DG, Pittenger MF, Stem Cells 2017, 35, 851; [PubMed: 28294454] e) Shih Y-R, Phadke A, Yamaguchi T, Kang H, Inoue N, Masuda K, Varghese S, Acta Biomater. 2015, 19, 1. [PubMed: 25805106]

[3] a). Brusatin G, Panciera T, Gandin A, Citron A, Piccolo S, Nat. Mater 2018, 17, 1063; [PubMed: 30374202] b) Webber MJ, Appel EA, Meijer EW, Langer R, Nat. Mater 2016, 15, 13; [PubMed: 26681596] c) Burdick Jason A., Mauck Robert L., Gerecht S, Cell Stem Cell 2016, 18, 13; [PubMed: 26748751] d) Rosales AM, Anseth KS, Nat. Rev. Mater 2016, 1, 15012; [PubMed: 29214058] e) Seale NM, Varghese S, J. Mater. Chem. B 2016, DOI: 10.1039/C5TB02658J;f) Lee K, Silva EA, Mooney DJ, J. R. Soc. Interface 2010, 8, 153. [PubMed: 20719768]

[4] a). Huangfu D, Maehr R, Guo W, Eijkelenboom A, Snitow M, Chen AE, Melton DA, Nat. Biotech 2008, 26, 795;b) Borowiak M, Maehr R, Chen S, Chen AE, Tang W, Fox JL, Schreiber SL, Melton DA, Cell Stem Cell 2009, 4, 348; [PubMed: 19341624] c) Kang H, Shih Y-RV, Nakasaki M, Kabra H, Varghese S, Sci. Adv 2016, 2, e1600691. [PubMed: 27602403]

[5] a). Fredholm B, Cell Death Differ. 2007, 14, 1315; [PubMed: 17396131] b) Haskó G, Linden J, Cronstein B, Pacher P, Nat. Rev. Drug Discovery 2008, 7, 759. [PubMed: 18758473]

[6]. Lopez CD, Bekisz JM, Corciulo C, Mediero A, Coelho PG, Witek L, Flores RL, Cronstein BN, Adv. Drug Deliv. Rev 2018, DOI: 10.1016/j.addr.2018.06.010.

[7] a). Carroll SH, Ravid K, Expert Rev. Mol. Med. 2013, 15;b) Ham J, Evans BA, Front Endocrinol. (Lausanne) 2012, 3, 113; [PubMed: 23024635] c) Cronstein BN, Sitkovsky M, Nat. Rev. Rheumatol 2017, 13, nrrheum.2016.178.

[8] a). Roszek K, Wujak M, J. Cell. Physiol 2019, 234, 320;b) Meling TR, Romundstad L, Niemi G, Narum J, Eide PK, Sorteberg AG, Sorteberg WA, Neurosurg. Rev 2018, 41, 585. [PubMed: 28819885]

[9] a). Biaggioni I, Olafsson B, Robertson RM, Hollister AS, Robertson D, Circ. Res 1987, 61, 779; [PubMed: 3677336] b) Kazemzadeh-Narbat M, Annabi N, Tamayol A, Oklu R, Ghanem A, Khademhosseini A, J. Drug Target 2015, 23, 580. [PubMed: 26453156]

[10] a). Ryu JH, Lee GJ, Shih Y, Kim T, Varghese S, Curr. Med. Chem 2018;b) Zhou Z, Zhang M, Liu Y, Li C, Zhang Q, Oupicky D, Sun M, Biomacromolecules 2018, DOI: 10.1021/ acs.biomac.8b00922;c) Brooks WLA, Sumerlin BS, Chem. Rev 2016, 116, 1375. [PubMed: 26367140]

[11] a). Amin S, Achenbach SJ, Atkinson EJ, Khosla S, Melton LJ, J. Bone Miner. Res 2014, 29, 581; [PubMed: 23959594] b) Haffner-Luntzer M, Kovtun A, Rapp AE, Ignatius A, Curr. Mol. Bio. 
Rep 2016, 2, 101;c) Burge R, Dawson-Hughes B, Solomon DH, Wong JB, King A, Tosteson A, J. Bone Miner. Res 2007, 22, 465. [PubMed: 17144789]

[12] a). Mediero A, Wilder T, Perez-Aso M, Cronstein BN, FASEB J 2015, 29, 1577; [PubMed: 25573752] b) Shih YRV, Liu MQ, Kwon SK, Iida M, Gong Y, Sangaj N, Varghese S, Sci. Adv 2019, 5.

[13] a). Shih Y-RV, Hwang Y, Phadke A, Kang H, Hwang NS, Caro EJ, Nguyen S, Siu M, Theodorakis EA, Gianneschi NC, Vecchio KS, Chien S, Lee OK, Varghese S, Proc. Natl. Acad. Sci 2014, 111, 990; [PubMed: 24395775] b) Carroll SH, Wigner NA, Kulkarni N, Johnston-Cox H, Gerstenfeld LC, Ravid K, J. Biol. Chem 2012, 287, 15718; [PubMed: 22403399] c) Kang H, Shih Y-RV, Varghese S, Biomacromolecules 2015, 16, 1050. [PubMed: 25686297]

[14] a). Kang H, Shih Y-RV, Hwang Y, Wen C, Rao V, Seo T, Varghese S, Acta Biomater 2014, 10, 4961; [PubMed: 25153779] b) Kang H, Wen C, Hwang Y, Shih Y-RV, Kar M, Seo SW, Varghese S, J. Mater. Chem. B 2014, 2, 5676. [PubMed: 25114796]

[15]. Kang H, Zeng Y, Varghese S, Acta Biomater 2018, 78, 365. [PubMed: 30031911]

[16]. Einhorn TA, Gerstenfeld LC, Nat. Rev. Rheumatol 2015, 11, 45. [PubMed: 25266456]

[17]. Baht GS, Nadesan P, Silkstone D, Alman BA, Bone 2017, 98, 31. [PubMed: 28254468]

[18] a). Antonioli L, Blandizzi C, Pacher P, Hasko G, Nat. Rev. Cancer 2013, 13, 842; [PubMed: 24226193] b) Montesinos MC, Desai A, Chen JF, Yee H, Schwarzschild MA, Fink JS, Cronstein BN, Am. J. Pathol 2002, 160, 2009. [PubMed: 12057906]

[19] a). Kusumbe AP, Ramasamy SK, Adams RH, Nature 2014, 507, 323; [PubMed: 24646994] b) Xu R, Yallowitz A, Qin A, Wu Z, Shin DY, Kim JM, Debnath S, Ji G, Bostrom MP, Yang X, Zhang C, Dong H, Kermani P, Lalani S, Li N, Liu Y, Poulos MG, Wach A, Zhang Y, Inoue K, Di Lorenzo A, Zhao B, Butler JM, Shim JH, Glimcher LH, Greenblatt MB, Nat. Med 2018, 24, 823. [PubMed: 29785024]

[20] a). Hwang Y, Zhang C, Varghese S, J. Mater. Chem 2010, 20, 345;b) Ayala R, Zhang C, Yang D, Hwang Y, Aung A, Shroff SS, Arce FT, Lal R, Arya G, Varghese S, Biomaterials 2011, 32, 3700. [PubMed: 21396708] 


\section{a}

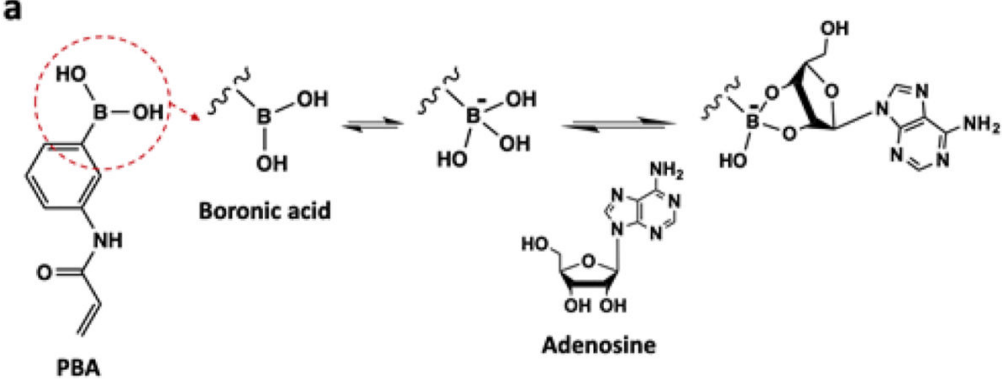

b

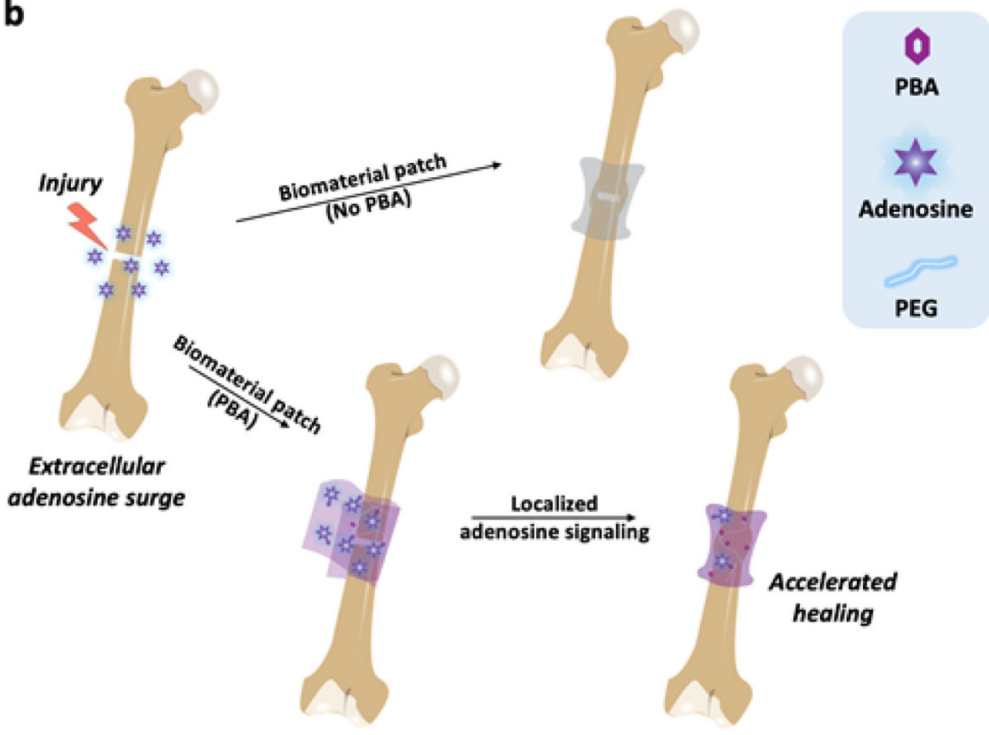

Figure 1. Schematics of PBA-mediated adenosine sequestration.

$\boldsymbol{a}$, 3-(acrylamido)phenylboronic acid (PBA) contains boronic acid moiety (circled in red), which forms a dynamic covalent complex of cyclic boronate ester with cis-diol-bearing adenosine at physiological pH. $\boldsymbol{b}$, PBA-based biomaterial patch sequesters extracellular adenosine at the fracture site while leveraging the adenosine surge after injury and sustains a localized adenosine signaling to accelerate tissue repair. 
a

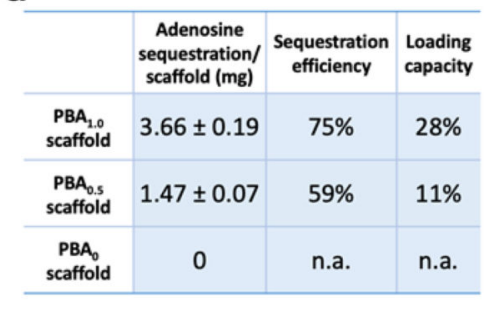

C

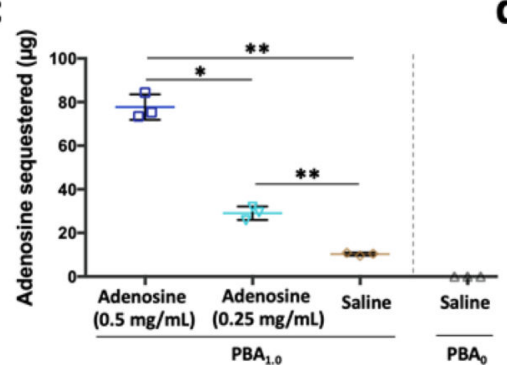

b

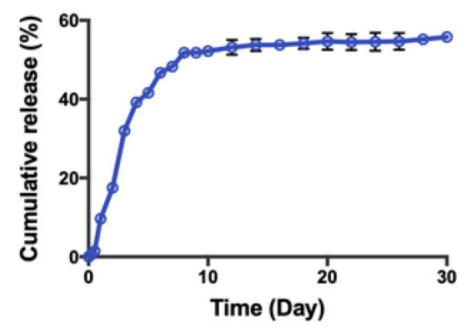

d

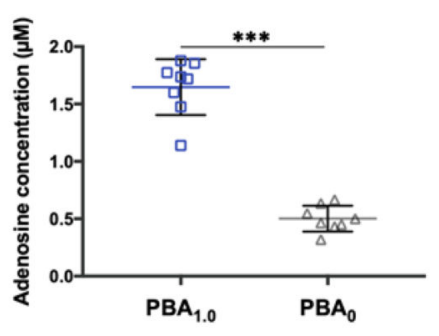

Figure 2. PBA scaffolds sequester adenosine both in vitro and in vivo.

$\boldsymbol{a}$, Table lists the amount of adenosine sequestered by each scaffold and the corresponding sequestration efficiency and loading capacity ( $\mathrm{n}=5$ scaffolds for each group). $\boldsymbol{b}$, Cumulative release of adenosine from the $\mathrm{PBA}_{1.0}$ scaffolds incubated in culture medium over $30 \mathrm{~d}(\mathrm{n}=3$ $\mathrm{PBA}_{1.0}$ scaffolds). $\boldsymbol{c}$, Amount of adenosine sequestered in each subcutaneously implanted scaffold following the injection of saline, $0.25 \mathrm{mg} / \mathrm{mL}$ and $0.5 \mathrm{mg} / \mathrm{mL}$ adenosine solution, respectively ( $\mathrm{n}=3$ scaffolds for each injection condition). $\boldsymbol{d}$, Scaffolds were excised from the fracture site at $3 \mathrm{~d}$ post implantation, and the sequestered adenosine was quantified ( $\mathrm{n}=8$ scaffolds for each group). All data are presented as means ( \pm s.d.). One-way ANOVA with Tukey's multiple-comparisons test was used for statistical analysis in $\boldsymbol{c}$; a two-tailed $\boldsymbol{t}$-test (unpaired) was used for $\boldsymbol{d}$. Significance is determined as $* P<0.05$, $* * P<0.01$, and $* * * P<$ 0.001 . 

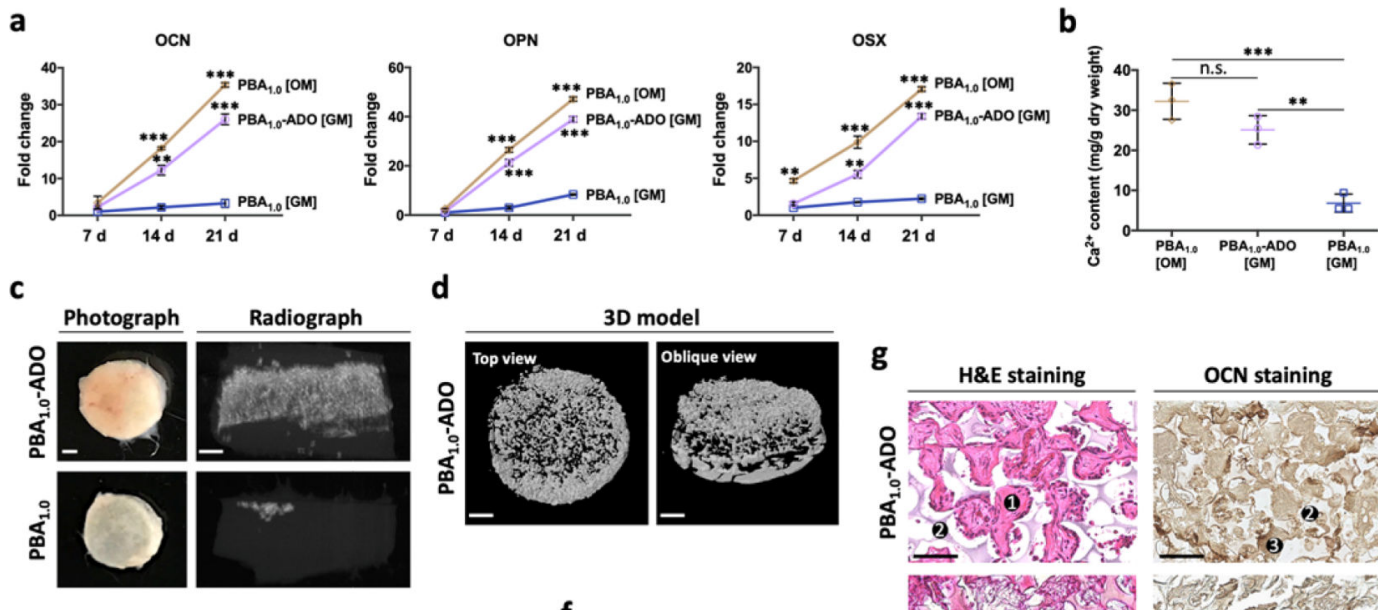

d

3D model
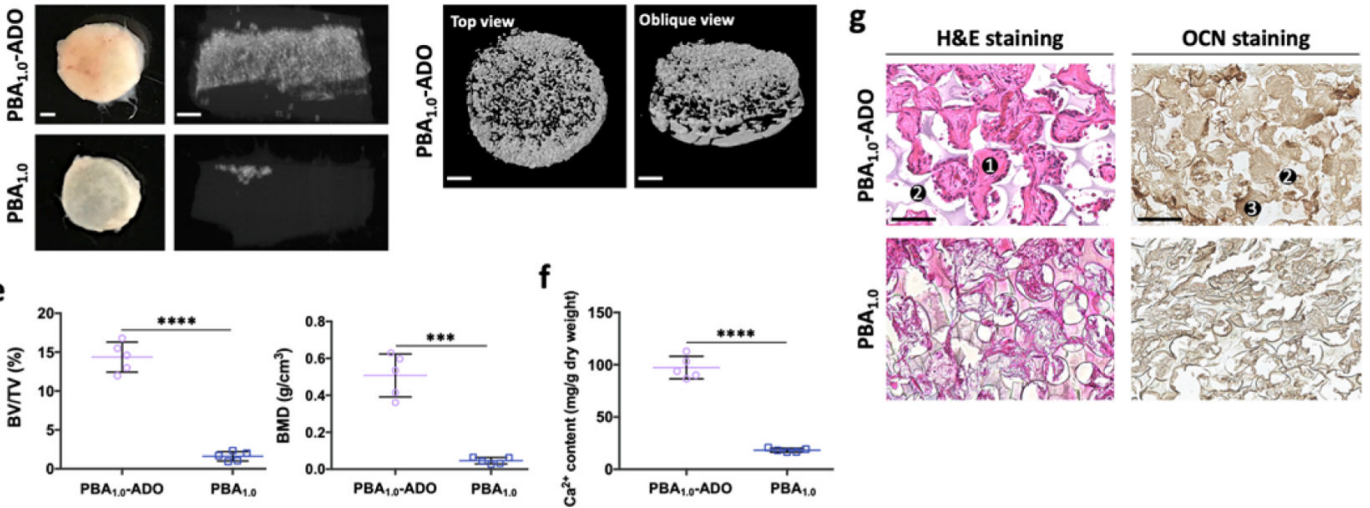

Figure 3. Adenosine-sequestered PBA scaffolds support osteogenic differentiation of hMSCs both in vitro and in vivo.

$\boldsymbol{a}, \mathrm{PBA}_{1.0}$ and $\mathrm{PBA}_{1.0}$-ADO scaffolds loaded with hMSCs were cultured in vitro for $21 \mathrm{~d}$.

Expression levels of osteogenic markers (OCN, OPN, and OSX) were quantified as a function of time and presented as fold change against $18 \mathrm{~s}$ levels $(n=3$ purified gene specimens for each group). GM: growth medium; OM: osteogenic-inducing medium. $\boldsymbol{b}$, Calcium content in each scaffold at $21 \mathrm{~d}(\mathrm{n}=3$ scaffolds for each group). $\boldsymbol{c}$, Representative photos and corresponding radiographs of the cell-laden $\mathrm{PBA}_{1.0}$ and $\mathrm{PBA}_{1.0}$-ADO scaffolds excised at Day 28 post subcutaneous implantation $(n=5$ scaffolds for each group). Scale bars, $1 \mathrm{~mm}$. $\boldsymbol{d}$, 3D reconstruction of a retrieved cell-laden $\mathrm{PBA}_{1.0}$-ADO scaffold shows the distribution of calcification, presented in both top view and oblique view. Scale bars, $1 \mathrm{~mm}$. $\boldsymbol{e}$, Bone volume ratio (BV/TV) and bone mineral density (BMD) of the retrieved scaffolds were quantified based on microcomputed tomography ( $\mathrm{n}=5$ scaffolds for each group). $f$, Calcium content in each scaffold at Day 28 ( $\mathrm{n}=5$ scaffolds for each group). $\boldsymbol{g}$, Representative microscopic images of $\mathrm{H} \& \mathrm{E}$ staining and OCN immunohistochemical staining within the retrieved scaffolds ( $\mathrm{n}=5$ scaffolds for each group). 1 , neo-bone tissue; 2 , scaffold; 3, OCN-positive tissue. Scale bars, $100 \mu \mathrm{m}$. All data are presented as means $( \pm$ s.d.). One-way ANOVA with Tukey's multiple-comparisons test was used for statistical analysis in $\boldsymbol{b}$; a two-tailed $\boldsymbol{t}$-test (unpaired) was used for $\boldsymbol{a}$ (with reference to the group of $\mathrm{PBA}_{1.0}[\mathrm{GM}]$ at $\left.7 \mathrm{~d}\right), \boldsymbol{e}$, and $\boldsymbol{f}$. Significance is determined as $* * P<0.01, * * * P<0.001$, $* * * * P<0.0001$, and n.s. (not significant). 
a

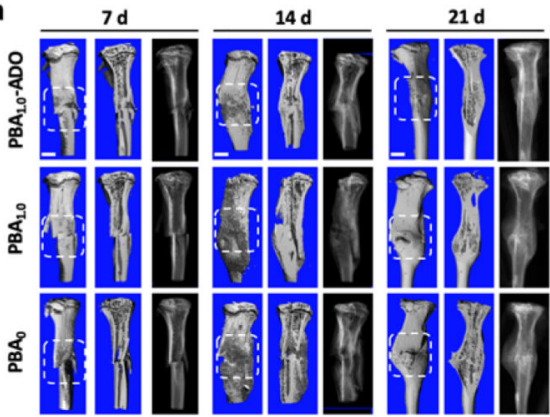

b
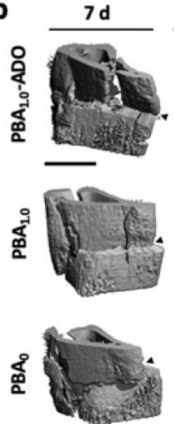
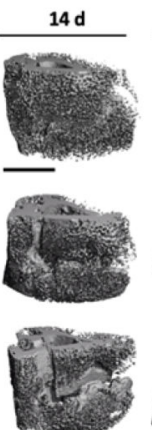
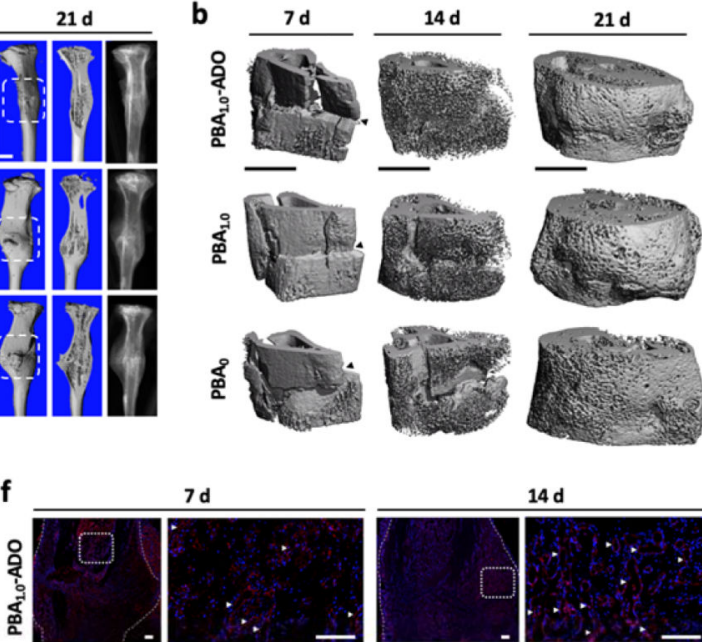

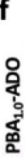
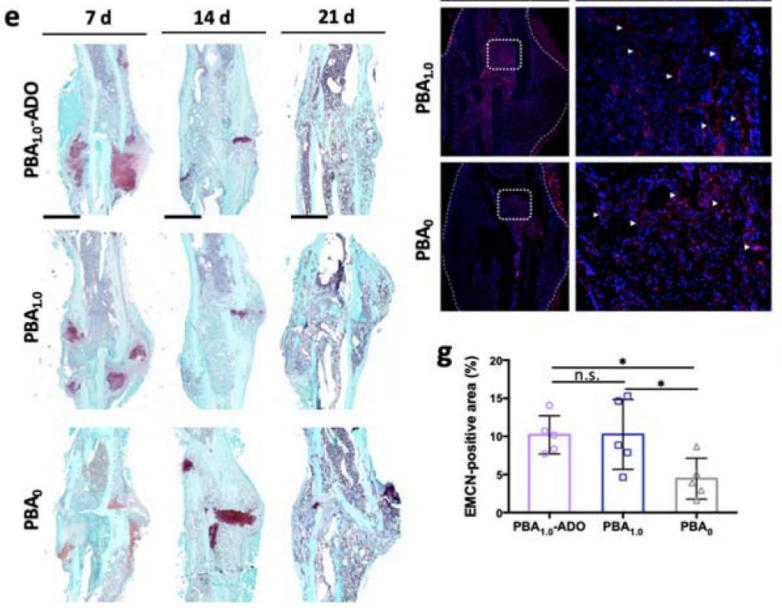

$14 \mathrm{~d}$
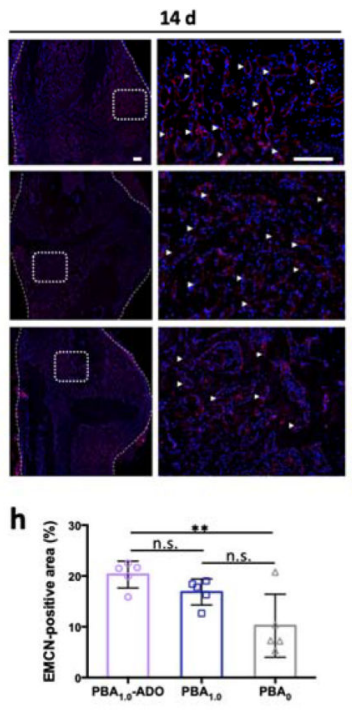

Figure 4. PBA-containing biomaterial patches facilitate callus maturation and promote fracture healing.

$a, 3 \mathrm{D}$ reconstructions (intact and cut views) and corresponding radiographs of the fractured tibiae treated with various biomaterial patches. Tissues were harvested at $7 \mathrm{~d}, 14 \mathrm{~d}$, or $21 \mathrm{~d}$ bars, $1 \mathrm{~mm}$. $\boldsymbol{b}$, Magnified 3D reconstructions of the callus regions show the evolution of
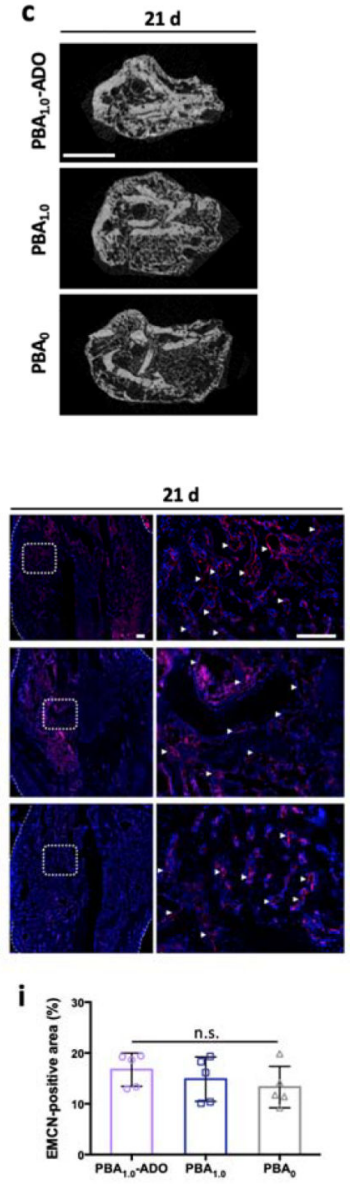
following injury ( $n=7$ mice for each treatment). White boxes cover the callus regions. Scale calluses during fracture healing with black arrowheads indicating fracture sites. Scale bars, 1 $\mathrm{mm}$. $\boldsymbol{c}$, Representative axial views of fracture sites show remodeling outcome within calluses by $21 \mathrm{~d}$. Scale bar, $1 \mathrm{~mm}$. $\boldsymbol{d}$, Bone volume ratio (BV/TV) of calluses at $21 \mathrm{~d}$ was quantified $\left(\mathrm{n}=7\right.$ mice for the cohort treated with $\mathrm{PBA}_{1.0}$-ADO, $\mathrm{n}=6$ mice for the cohort treated with $\mathrm{PBA}_{1.0}$ or $\left.\mathrm{PBA}_{0}\right)$. $\boldsymbol{e}$, Representative safranin-O staining images of fractured tibiae treated with various biomaterial patches show the evolution of cartilaginous tissues (red) in calluses ( $\mathrm{n}=7$ mice for each treatment). Scale bars, $1 \mathrm{~mm}$. $f$, Representative immunofluorescence images of endomucin (EMCN, red) show the vascularization in calluses $(n=7$ mice for each treatment). Nuclei were stained with DAPI (blue). White dashed lines delineate the callus boundary. Magnified images of the region covered in white boxes are displayed with white arrowheads indicating EMCN-positive vessels. Scale bars, $200 \mu \mathrm{m} . \boldsymbol{g}, \boldsymbol{h}, \boldsymbol{i}$, Quantification of the relative EMCN-positive vessel area to the callus area at $7 \mathrm{~d}(\boldsymbol{g}), 14 \mathrm{~d}(\boldsymbol{h})$, and $21 \mathrm{~d}(\boldsymbol{i})$ based on immunofluorescence images $(n=5$ mice from each treatment; each data point is 
averaged from 5 images for each mouse). In $\boldsymbol{d}$ and $\boldsymbol{g}-\boldsymbol{i}$, data are presented as means ( \pm s.d.). One-way ANOVA with Tukey's multiple-comparisons test was used for statistical analysis in $\boldsymbol{d}$ and $\boldsymbol{g}-\boldsymbol{i}$. Significance is determined as $* P<0.05$, $* * P<0.01, * * * P<0.001$, and n.s. (not significant). 